\title{
Two-Dimensional Diffusion of Excitons in a Perylene Diimide Monolayer Quenched by a Fullerene Heterojunction
}

\author{
James Kerfoot, ${ }^{* \dagger}{ }^{\dagger}$ Vladimir V. Korolkov, ${ }^{\dagger}$ Simon A. Svatek, ${ }^{\dagger, \perp}$ Manal Alkhamisi, ${ }^{\dagger}$ Takashi Taniguchi, ${ }^{\ddagger}$ \\ Kenji Watanabe, ${ }^{\ddagger}$ Patrick W. Parkinson, ${ }^{\S, \|(0)}$ and Peter H. Beton ${ }^{\dagger}$ (1) \\ ${ }^{\dagger}$ School of Physics \& Astronomy, University of Nottingham, Nottingham NG7 2RD, U.K. \\ ${ }^{*}$ National Institute for Materials Science, 1-1 Namiki, Tsukuba, Ibaraki 305-0044, Japan \\ ${ }^{\S}$ School of Physics and Astronomy and "Photon Science Institute, University of Manchester, Oxford Road, Manchester M13 9PL, \\ U.K.
}

\section{Supporting Information}

ABSTRACT: The structural and functional properties of organic heterojunctions play a vitally important role in the operation of organic devices, but their properties are difficult to measure directly due to the buried interfaces that are typically formed. We have grown model heterojunctions consisting of two monolayer-thick organic semiconductors and used these bilayers to explore the two-dimensional dynamics of excitons. The heterostructures are formed by sequential deposition of monolayers of perylene-3,4,9,10-tetracarboxylic$3,4,9,10$-diimide (PTCDI) and fullerene $\left(\mathrm{C}_{60}\right)$ on hexagonal

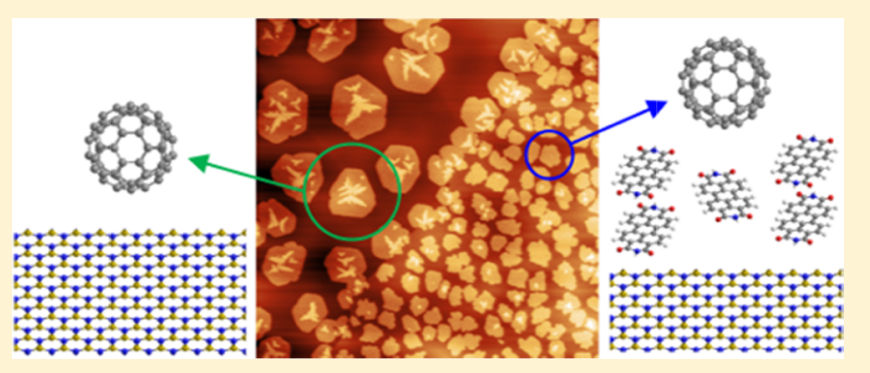
boron nitride $(\mathrm{hBN})$. The morphology of these bilayers was characterized using atomic force microscopy and showed clear differences in the $\mathrm{C}_{60}$ growth kinetics on $\mathrm{hBN}$ and PTCDI. The variation in the fluorescence of PTCDI-C 60 heterostructures with increasing fullerene coverage showed a reduction in intensity consistent with exciton diffusion and quenching. We use a simple model for the intensity dependence to determine the two-dimensional exciton diffusion length in a PTCDI monolayer, finding a value of $17 \pm 3 \mathrm{~nm}$ for this parameter.

\section{INTRODUCTION}

Heterostructure formation by the sequential deposition of distinct molecular species represents a promising route to engineer the functional properties of organic devices as well as enabling the growth of model heterointerfaces. ${ }^{1-6}$ Organic heterostructures are typically characterized at the molecular scale using scanning tunneling microscopy, and although this has been extremely successful, the requirement for conductive surfaces limits compatibility with subsequent optical measurements. ${ }^{7,8}$ This motivates the formation of analogue heterostructures on insulating surfaces, on which both the morphology and functional properties of organic films may be measured and compared systematically. ${ }^{9-12}$ We have recently demonstrated that atomic force microscopy (AFM) can be used under ambient conditions to acquire images of molecules on an insulating surface with submolecular resolution, allowing the correlation of supramolecular order and optical properties. Specifically, we have shown that the fluorescence of monolayer (ML)-thick supramolecular assemblies formed on the hexagonal boron nitride $(\mathrm{hBN})$ surface may be measured ${ }^{13-15}$ and, in a separate study, that epitaxial supramolecular heterostructures may also be formed on $\mathrm{hBN}$. $^{2}$ In this paper, we explore the fluorescence of model heterostructures formed by the sequential growth of monolayers of two organic semiconductors through the deposition of $\mathrm{C}_{60}$ on preformed supramolecular arrays of perylene-3,4,9,10-tetracarboxylic-diimide (PTCDI), thus forming a bilayer heterojunction. The fullerene islands quench fluorescence, and we show that the dependence of emission intensity on coverage provides an insight into the transport of excitons within the PTCDI monolayer. This represents a lowdimensional analogue of experiments in which bulk heterostructures composed of a fluorophore and an exciton quenching layer, typically fullerene, are used to determine exciton diffusion lengths. ${ }^{16-19}$ In our case, the geometry of the heterostructures allows the decoupling of exciton diffusion in different directions and we are able to measure the twodimensional diffusion length of excitons in PTCDI parallel to the molecular plane.

\section{METHODS}

Substrates were prepared using the scotch tape method to mechanically exfoliate $\mathrm{hBN}$ crystals onto a $\mathrm{Si}$ wafer on which a $\mathrm{SiO}_{2}$ layer had been grown thermally, as described in our earlier work. ${ }^{13-15}$ To deposit molecular films, clean substrates were loaded into a vacuum chamber (base pressure $10^{-8} \mathrm{mbar}$ )

Received: February 13, 2019

Revised: April 24, 2019

Published: April 25, 2019 
and annealed at approximately $450{ }^{\circ} \mathrm{C}$ prior to the deposition of PTCDI and $\mathrm{C}_{60}$ from two Knudsen cells, which were heated to 442 and $374{ }^{\circ} \mathrm{C}$, respectively. The deposition rate of each species was determined using a quartz crystal microbalance and calibrated from AFM measurements of thick films of each molecular species. The temperature of the $\mathrm{hBN}$ on $\mathrm{SiO}_{2}$ substrates during growth was controlled using a type $\mathrm{C}$ thermocouple and a tantalum heating element. After deposition, the chamber was vented to nitrogen and samples were removed for characterization under ambient conditions using AFM and fluorescence spectroscopy.

To characterize the sample morphology, AFM measurements were carried out using the Asylum Research Cypher S atomic force microscope with Multi75AL-G silicon cantilevers from Budges Sensors. Fluorescence measurements were acquired using the HORIBA MicOS fluorescence spectrometer with a $405 \mathrm{~nm}$ delta diode excitation source. The pulse rate of the delta diode excitation source was $100 \mathrm{MHz}$, with a typical pulse width of 45 ps. The excitation source was used in combination with a clean-up (405 nm longpass) filter, which reduced the average power from its maximum $(1.4 \mathrm{~mW})$ to less than $100 \mu \mathrm{W}$. To eliminate the effect of optical interference in the photoluminescence intensity, with a more detailed description given in the Supporting Information (SI), all fluorescence measurements were acquired from few-layer $\mathrm{hBN}$ flakes, identified through their optical contrast on $90 \mathrm{~nm}$ $\mathrm{SiO}_{2}$ substrates.

\section{RESULTS AND DISCUSSION}

The growth of both PTCDI and $\mathrm{C}_{60}$ is carried out by sublimation in vacuum and studied using AFM under ambient conditions, as described above. Figure 1 shows the morphology of sub-monolayer coverages of each component molecule PTCDI (Figure $1 \mathrm{a}-\mathrm{c}$ ) and $\mathrm{C}_{60}$ (Figure $\left.1 \mathrm{~d}, \mathrm{e}\right)$. Figure 1c shows needlelike islands with monolayer height formed following the deposition of 0.3 monolayers (ML) of PTCDI on a hBN substrate held at $135{ }^{\circ} \mathrm{C}$. The average length and areal density of PTCDI islands were $6.4 \pm 1.5 \mu \mathrm{m}$ and $0.027 \pm 0.008 \mu \mathrm{m}^{-2}$, respectively. High-resolution images show that PTCDI forms a canted packing structure with inter- $\left(a_{1}\right)$ and intra- $\left(a_{2}\right)$ row lattice vectors $a_{1}=1.74 \pm 0.10 \mathrm{~nm}$ and $a_{2}=1.48 \pm 0.10 \mathrm{~nm}$ subtended by an angle $\gamma=87 \pm 2^{\circ}$ (see Figure 1a,b), in agreement with the previous work. ${ }^{14}$ In contrast to PTCDI monolayers grown on alkali-halide substrates, where molecular dewetting leads to a reorganization of the film structure, ${ }^{20-22}$ PTCDI monolayers were found to be stable on hBN. The lateral dimensions of the islands show a strong dependence on substrate temperature; results for a range of substrate temperatures are shown in the SI.

At sub-monolayer $(0.3 \mathrm{ML})$ coverage, when grown at room temperature, $\mathrm{C}_{60}$ was found to form monolayer islands of irregular shape with second layers also observed (see Figure 1d). The presence of monolayers was confirmed by an island step height measurement of $0.8 \pm 0.1 \mathrm{~nm}$, corresponding to one monolayer. From high-resolution AFM images, $\mathrm{C}_{60}$ was found to arrange in a hexagonal packing structure, with a lattice constant of $1.0 \pm 0.1 \mathrm{~nm}$, in good agreement with $\mathrm{C}_{60}$ thin films deposited on both conductive and insulating substrates $^{23-26}$ (see Figure 1d inset). From large area AFM images, the average size and areal density of $\mathrm{C}_{60}$ islands grown at room temperature were found to be $0.46 \pm 0.07 \mu \mathrm{m}$ and 2.9 $\pm 0.2 \mu \mathrm{m}^{-2}$, respectively. As the growth temperature of $\mathrm{C}_{60}$ was increased, the size of monolayer islands increased while
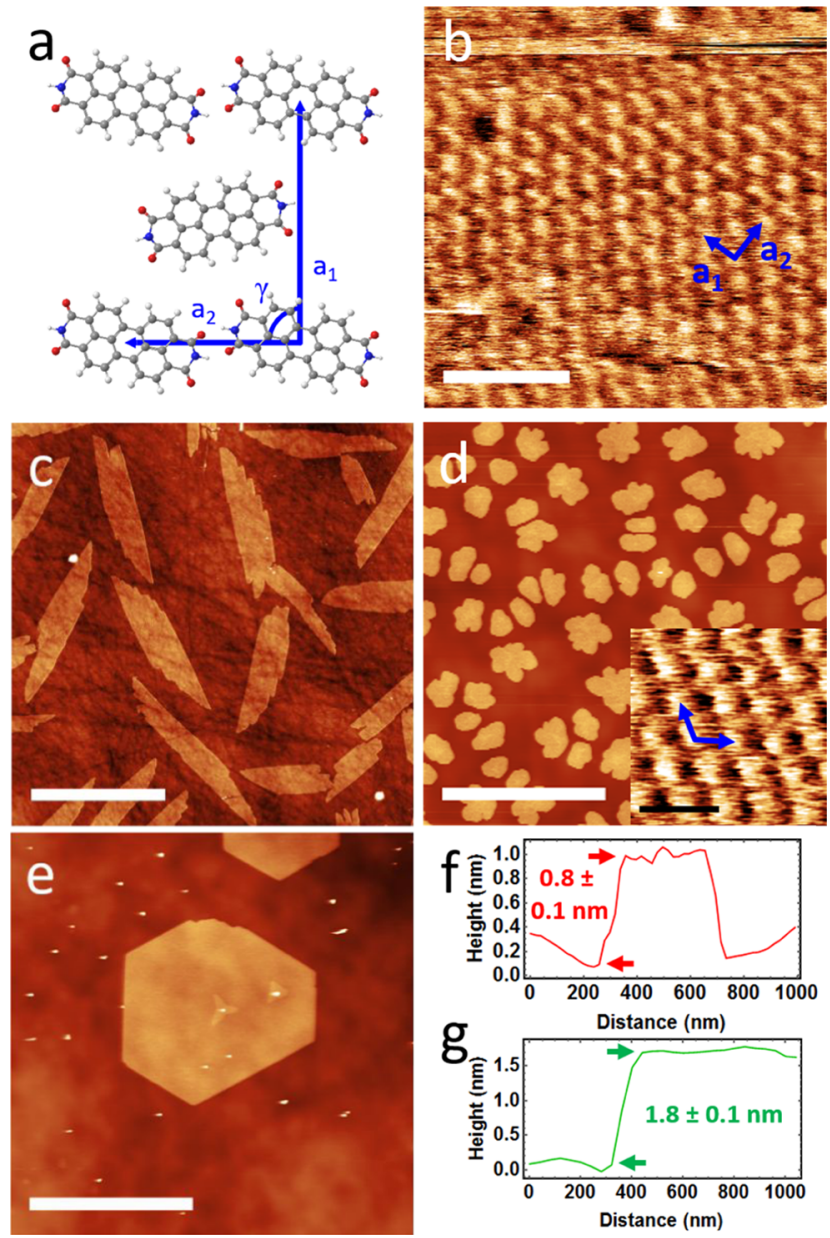

Figure 1. (a) Schematic of molecular structure and canted arrangement of PTCDI molecules on hBN is shown; (b) highresolution AC mode AFM image (scale bar $5 \mathrm{~nm}$ ) of PTCDI monolayers on $\mathrm{hBN}$ showing molecules arranged in the canted structure; (c) larger area AFM image (scale bar $10 \mu \mathrm{m}$ ) of PTCDI grown $(0.3 \mathrm{ML})$ on $\mathrm{hBN}$ at $135{ }^{\circ} \mathrm{C}$ showing needlelike islands; (d) AFM images of a sub-monolayer $(0.3 \mathrm{ML})$ coverage of $\mathrm{C}_{60}$ deposited at room temperature (scale bar $2 \mu \mathrm{m}$ ) with a high-resolution image (scale bar $2 \mathrm{~nm}$ ) inset; (e) sub-monolayer coverage of $\mathrm{C}_{60}$ deposited at $216{ }^{\circ} \mathrm{C}$ (scale bar $2 \mu \mathrm{m}$ ); representative height profiles taken from samples grown at $21{ }^{\circ} \mathrm{C}(\mathrm{f})$ and $216^{\circ} \mathrm{C}(\mathrm{g})$ confirm a change from monolayer to bilayer growth.

the island density decreased and at a growth temperature of $216{ }^{\circ} \mathrm{C}$ only faceted bilayer islands were observed; see Figure 1e. Further results showing the dependence of the morphology of $\mathrm{C}_{60}$ islands on coverage and substrate temperature are included in SI.

The transition from monolayer to bilayer $\mathrm{C}_{60}$ islands has been reported previously in studies of growth on other insulating substrates. ${ }^{21-24,27-31}$ However, the transition to bilayer growth occurs at a higher growth temperature $\left(178^{\circ} \mathrm{C}\right)$ on $\mathrm{hBN}$ than on the alkali-halide substrates explored in the literature. The observed transition to bilayers at higher temperatures as well as the Arrhenius-type behavior of the island density discussed in the SI suggests that the growth of $\mathrm{C}_{60}$ on hBN is kinetically limited. ${ }^{22,32,33}$

Having established the morphology of both PTCDI and $\mathrm{C}_{60}$ on hBN, molecular heterostructures were formed by the sequential deposition of the two species. A $0.3 \mathrm{ML}$ coverage of PTCDI was first deposited onto hBN substrates held at 
elevated temperatures $\left(135{ }^{\circ} \mathrm{C}\right)$ to form monolayer islands much larger than the typical interisland separation of $\mathrm{C}_{60}$ on $\mathrm{hBN}$; note also that these samples have exposed $\mathrm{hBN}$ between the PTCDI islands, allowing a direct comparison of the growth of $\mathrm{C}_{60}$ on PTCDI and hBN. The substrate was then cooled to room temperature prior to the deposition of $0.3 \mathrm{ML}$ of $\mathrm{C}_{60}$ from a second Knudsen cell. The growth of $\mathrm{C}_{60}$ on $\mathrm{hBN}$ and PTCDI is strikingly different (Figure 2a,b); the islands grown on PTCDI (marked by a blue arrow in Figure $2 b$ ) are of monolayer height but are much smaller and have higher areal density than those growing on the exposed hBN (marked by a green arrow). Specifically, the island density on PTCDI ( $45 \pm$ $\left.8 \mu \mathrm{m}^{-2}\right)$ was an order of magnitude higher than on hBN (4.7
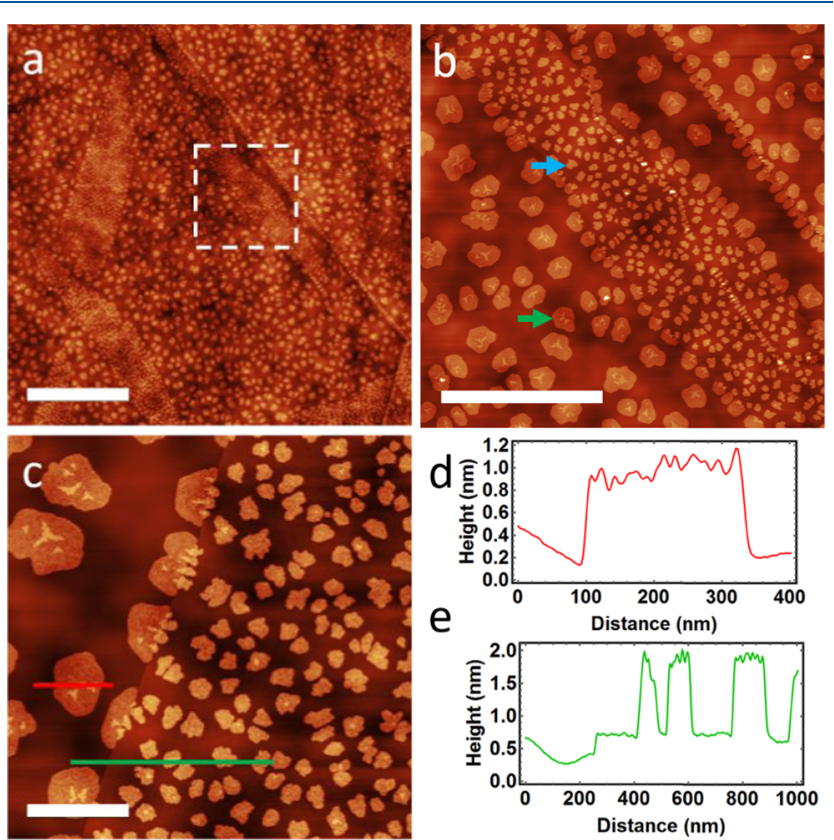

e

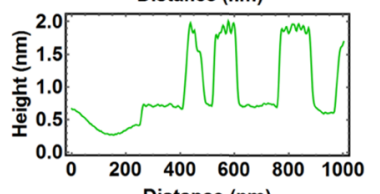

Distance $(\mathrm{nm})$
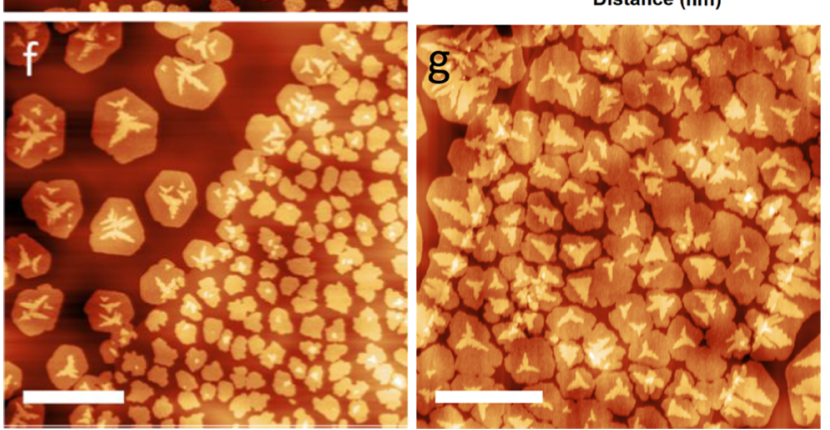

Figure 2. (a) Large area AFM images (scale bar $5 \mu \mathrm{m}$ ) showing the island morphology of the heterostructures formed by depositing 0.3 ML of PTCDI followed by $0.3 \mathrm{ML}$ of $\mathrm{C}_{60}$; PTCDI islands are identifiable by the increased island density of $\mathrm{C}_{60}$ over their surface; (b) higher magnification image (scale bar, $2 \mu \mathrm{m}$ ), indicated in (a), of the same sample reveals the island morphology of $\mathrm{C}_{60}$ on top of PTCDI (highlighted by blue arrow) and hBN (green arrow). (c) AFM image (scale bar, $500 \mathrm{~nm}$ ) showing the morphology of PTCDI$\mathrm{C}_{60}$ bilayer heterostructures on $\mathrm{hBN}$ with corresponding line profiles in red (d) and green (e), with step heights of $0.3 \pm 0.1$ and $0.8 \pm 0.1$ $\mathrm{nm}$, respectively, confirming the presence of monolayers of both PTCDI (0.3 ML) and $\mathrm{C}_{60}$. AFM images (scale bars of both, $500 \mathrm{~nm}$ ) of samples with higher coverages of $\mathrm{C}_{60}-0.8 \mathrm{ML}(\mathrm{f})$ and $1.2 \mathrm{ML}(\mathrm{g})$ are also shown. In each of the images (c), (f), and $(\mathrm{g})$, the step edge of PTCDI runs diagonally across the image, with areas of uncovered $\mathrm{hBN}$ present in the upper left. $\left.\pm 0.3 \mu \mathrm{m}^{-2}\right)$. Nevertheless, the overall coverage of $\mathrm{C}_{60}$ was the same on the PTCDI monolayer and the surrounding hBN layer implying that the diffusion length of $\mathrm{C}_{60}$ is small compared with the dimensions of the PTCDI islands.

The heterostructure islands in Figure $1 \mathrm{~b}$ differ from the morphology previously observed for PTCDI and $\mathrm{C}_{60}$ codeposited on calcium fluoride $\left(\mathrm{CaF}_{2}\right)$, where dewetting from the substrate leads to smaller multilayered PTCDI islands with more complicated island morphologies. ${ }^{34}$ A significant difference in our work is the much larger, flat monolayer PTCDI islands that can be formed on $\mathrm{hBN}$; these allow the kinetically limited growth of $\mathrm{C}_{60}$ islands without a dominant contribution to nucleation from sites such as PTCDI step edges.

AFM images of PTCDI/ $\mathrm{C}_{60}$ heterostructures in regions close to PTCDI island edges (see Figure 2c) reveal a similar morphology to that in Figure 1, as indicated by the height profiles in Figure 2d,e, which confirms the monolayer height of $\mathrm{C}_{60}$ on PTCDI (in Figure $2 \mathrm{c}, \mathrm{f}, \mathrm{g}$, the upper left shows regions of $\mathrm{C}_{60}$ growth on $\mathrm{hBN}$ whereas the lower right shows the PTCDI $/ \mathrm{C}_{60}$ heterostructure). Interestingly, the $\mathrm{C}_{60}$ islands are present on both sides of the step edges of the PTCDI monolayer, with second layers of $\mathrm{C}_{60}$ that appear to grow across the edge of the PTCDI island. In addition, as the $\mathrm{C}_{60}$ thickness is increased, we observe many areas of the second layer $\mathrm{C}_{60}$. For a given coverage (e.g., Figure $2 \mathrm{f}$ ), the second layers were more prevalent on islands growing on the hBN (Figure $2 \mathrm{f}$ upper left) than on PTCDI (lower right). This suggests that on the smaller fullerene islands formed on PTCDI, incident $\mathrm{C}_{60}$ molecules may diffuse to the island edge and undergo thermally activated downward hopping; however, on the larger islands formed on $\mathrm{hBN}$, nucleation of a second $\mathrm{C}_{60}$ layer occurs. This implies that the diffusion length of $\mathrm{C}_{60}$ on $\mathrm{C}_{60}$ lies in the range of the typical island dimensions on $\mathrm{hBN}(460 \pm 70 \mathrm{~nm})$ and PTCDI $(180 \pm 20 \mathrm{~nm})$.

To investigate the fluorescence of heterostructures, a set of samples were prepared with progressively thicker films (0$0.92 \mathrm{ML})$ of $\mathrm{C}_{60}$ overgrown on PTCDI $(0.93 \mathrm{ML})$. The fluorescence of sublimed PTCDI monolayers was measured using a $405 \mathrm{~nm}$ pulsed excitation source (photon energy $E_{\mathrm{ex}}=$ $3.06 \mathrm{eV}$, spot size $\sim 2 \mu \mathrm{m}$; see the SI). The spectra (Figure $3 \mathrm{a}$ ) show a strong $0-0$ peak at $2.208 \pm 0.002 \mathrm{eV}$ and a $0-1$ satellite peak at $2.045 \pm 0.002 \mathrm{eV}$, in good agreement with PTCDI films deposited on hBN from solution. ${ }^{.4}$ These peaks were clearly resolved in the spectra of $\mathrm{C}_{60}$-PTCDI heterostructures but with an intensity that progressively decreased with increasing coverage of $\mathrm{C}_{60}$. To eliminate errors due to optical interference, all fluorescence measurements were taken from few-layer optically thin hBN flakes, which were easily identifiable by the optical contrast of both the hBN flake on 90 $\mathrm{nm} \mathrm{SiO}_{2}$ and the adsorbed PTCDI monolayers (the dependence of the spectra on $\mathrm{hBN}$ flake thickness is discussed in the SI). Figure $3 a$ shows spectra averaged over several thin hBN flakes. Errors due to misalignment of the PTCDI islands with the laser spot were avoided since the PTCDI islands could be resolved using optical microscopy (see the SI).

The peak intensity for varying $C_{60}$ coverage is shown in Figure $3 \mathrm{~b}$ and does not follow a linear dependence, as might be expected were the PTCDI fluorescence simply quenched by the overlying $\mathrm{C}_{60}$ islands. Instead, our data is consistent with an effective quenching area that exceeds the physical island size due to the capture of excitons that are excited remotely and then diffuse laterally to the boundary of the $\mathrm{C}_{60}$ island. We approximate $\mathrm{C}_{60}$ islands as nonoverlapping regular circles of 

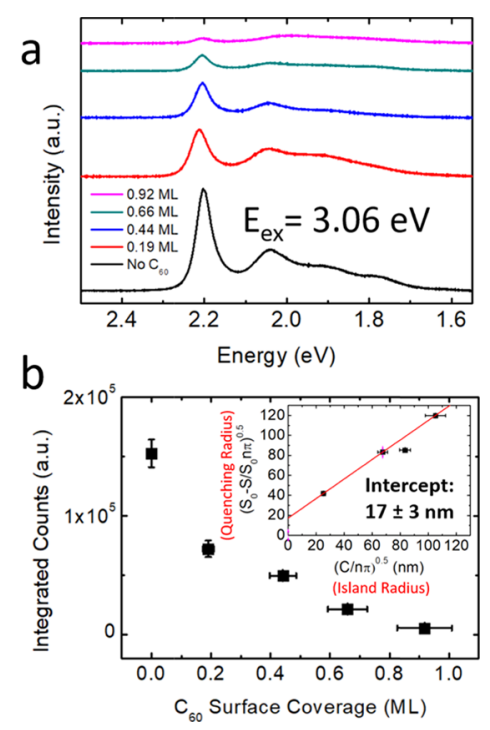

Figure 3. (a) Fluorescence spectra of PTCDI and PTCDI- $\mathrm{C}_{60}$ heterostructures, (the coverage of PTCDI was maintained at $0.93 \pm$ $0.05 \mathrm{ML})$ acquired from thin $\mathrm{hBN}$ flakes $(<2 \mathrm{~nm})$ were averaged over multiple flakes and are shown for a series of $\mathrm{C}_{60}$ coverages up to 0.92 ML. (b) The total integrated count of the $0-0$ peak of PTCDI (2.208 $\mathrm{eV}$ ) was extracted from the Lorentzian fitting and is plotted against the absolute $\mathrm{C}_{60}$ coverage measured using AFM. Taking island densities and coverages extracted from AFM images, the average island radius $(C / n \pi)^{0.5}$ is shown plotted against the effective radius over which PTCDI is quenched $\left(S_{0}-S / S_{0} n \pi\right)^{0.5}$, from which an exciton diffusion length of $17 \pm 3 \mathrm{~nm}$ was extracted under the assumptions stated in the main text ((b) inset).

radius, $R=(C / \pi n)^{1 / 2}$, for a given surface coverage, $C$, and island density, $n$. The effective capture radius is therefore given by $R+\delta$, where $\delta$ is the diffusion length. The fluorescence intensity, $S$, is therefore given by, $S=S_{0}\left(1-\pi n(R+\delta)^{2}\right)$. Figure $3 \mathrm{~b}$ inset shows the expected linear dependence $\left(\left(S_{0}-\right.\right.$ $\left.S) / S_{0} \pi n\right)^{1 / 2}=R+\delta$, obtained by rearranging the intensity dependence above. The intercept of the linear fit corresponds to a diffusion length $\delta=17 \pm 3 \mathrm{~nm}$ (we have excluded the 0.93 ML coverage of $\mathrm{C}_{60}$ when extracting our estimate for the exciton diffusion length since the model is not valid for coverages approaching $1 \mathrm{ML}$ due to island merger).

It is interesting to compare the diffusion length determined from our analysis to the published value, $9.9 \mathrm{~nm}$ for PTCDI$\mathrm{C} 8,{ }^{17}$ an alkylated derivative of PTCDI. In this previous study, a PTCDI-C8 thin film was overgrown on quaterterrylene and excitons were quenched at the interface. This lower value may reflect the fact that excitons need to diffuse across a barrier formed by solubilizing alkyl chains to access the quaterterrylene interface. The three-dimensional structure adopted by molecules in this previous experiment results in a more complex coupling of electronic and vibrational modes and the associated $\pi$-stacked structures can lead to other possible excitations, such as charge-transfer excitons and excimers. These processes can influence both diffusion of excitons and the photoluminescence of organic crystals and thick films ${ }^{35}$ compared with both isolated molecules and monolayers at interfaces. ${ }^{10,36}$ Interestingly, the structural organization inherent in the monolayers formed in our model heterostructures provides a route to decouple different modes of exciton diffusion; in the present case, the diffusion is parallel to the planes of the adsorbed molecules. Similar measurements for thin films of the closely related molecule perylene-3,4,9,10- tetracarboxylic dianhydride (PTCDA) $)^{18,19}$ yield values of the diffusion length in the range $10-15 \mathrm{~nm}$.

Our experiments do not provide direct information about the mechanism for exciton/charge transfer between PTCDI and fullerene, which results in quenching. However, in our experiments, we are able to confirm using AFM that PTCDI and fullerene molecules are nearest neighbors and so it is conceivable that ultrafast charge transfer between the highest occupied molecular orbital (HOMO) of the two molecules could be responsible for exciton quenching, ${ }^{37}$ in addition to the Förster mechanism, which occurs due to exciton transfer. Such effects would require a favorable alignment of the molecular orbitals of both species, which is suggested by values available in the literature, ${ }^{1,38-41}$ although it is important to note that these values serve only as a guide, since structural differences between isolated molecules, bulk crystals, and heterojunctions and molecules adsorbed on surfaces would be expected to lead to modest shifts in the energies of HOMO and lowest unoccupied molecular orbital levels (LUMO).

\section{CONCLUSIONS}

The formation of controlled heterostructures formed by the sequential deposition of monolayers of different molecular species opens up new directions in the research of the growth mechanisms of organic heterojunctions and their fundamental properties. The use of high-resolution AFM provides direct information about the molecular scale structure and growth of organic thin films of relevance to heterojunctions in organic devices and in this work has been used to determine the growth morphology of model interfaces that we show can be used to explore exciton diffusion in two dimensions. For the monolayer considered here, diffusion is parallel to the interface and the plane of the molecular $\pi$ system; however, it is possible to envisage the formation of monolayers of molecules with an upright geometry, thus allowing a systematic study of the relationship of geometry and structural organization to exciton diffusion. We extract an effective length of $17 \pm 3 \mathrm{~nm}$, over which excitons within the PTCDI monolayer can migrate to the perimeter of $\mathrm{C}_{60}$ islands. Although this extracted value is similar to those of other organic systems, it is noteworthy that the value we obtain is still relatively large given the lack of $\pi$ stacking within the PTCDI monolayer. This work highlights the possibility of exploring exciton diffusion and quenching in a much wider variety of heterojunctions, including those based on semiconducting polymers of relevance to photovoltaic and light-emitting devices, and plan to explore these materials in our future work.

\section{ASSOCIATED CONTENT}

\section{Supporting Information}

The Supporting Information is available free of charge on the ACS Publications website at DOI: 10.1021/acs.jpcc.9b01413.

Temperature- and coverage-dependent growth of neat films of PTCDI and $\mathrm{C}_{60}$ on $\mathrm{hBN}$, in addition to Arrhenius plots of the island area densities; data showing the effect of optical interference, arising from the thickness of the underlying hBN flake, on the fluorescence of PTCDI on hBN; AFM images of the morphology of $\mathrm{C}_{60} / \mathrm{PTCDI}$ heterostructures, from which island statistics were extracted and used in the interpretation of optical data (PDF) 


\section{AUTHOR INFORMATION}

\section{Corresponding Author}

*E-mail: James.Kerfoot@nottingham.ac.uk.

\section{ORCID}

James Kerfoot: 0000-0002-6041-4833

Patrick W. Parkinson: 0000-0001-9429-9768

Peter H. Beton: 0000-0002-2120-8033

\section{Present Address}

${ }^{\perp}$ Instituto de Energía Solar, Universidad Politécnica de Madrid, Madrid 28040, Spain (S.A.S.).

\section{Notes}

The authors declare no competing financial interest.

Original data files containing raw AFM images and fluorescence spectra are available from the University of Nottingham Research Data Management Service, DOI: $10.17639 /$ nott.6188.

\section{ACKNOWLEDGMENTS}

This work was supported by the Engineering and Physical Sciences Research Council [grant numbers EP/N033906/1, $\mathrm{EP} / \mathrm{P019080/1}$ ] and the Leverhulme Trust [grant number RPG-2016-104]. K.W. and T.T. acknowledge support from the Elemental Strategy Initiative conducted by the MEXT, Japan and the CREST (JPMJCR15F3), JST.

\section{REFERENCES}

(1) Schmitz-Hübsch, T.; Sellam, F.; Staub, R.; Förker, M.; Fritz, T.; Kübel, C.; Müllen, K.; Leo, K. Direct observation of organic-organic heteroepitaxy: perylene-tetracarboxylic-dianhydride on hexa-peribenzocoronene on highly ordered pyrolytic graphite. Surf. Sci. 2000, $445,358-367$.

(2) Korolkov, V. V.; Baldoni, M.; Watanabe, K.; Taniguchi, T.; Besley, E.; Beton, P. H. Supramolecular heterostructures formed by sequential epitaxial deposition of two-dimensional hydrogen-bonded arrays. Nat. Chem. 2017, 9, 1191.

(3) Chen, W.; Qi, D.-C.; Huang, H.; Gao, X.; Wee, A. T. S. Organic-organic heterojunction interfaces: effect of molecular orientation. Adv. Funct. Mater. 2011, 21, 410-424.

(4) Schwarze, M.; Tress, W.; Beyer, B.; Gao, F.; Scholz, R.; Poelking, C.; Ortstein, K.; Günther, A. A.; Kasemann, D.; Andrienko, D.; et al. Band structure engineering in organic semiconductors. Science 2016, 352, 1446.

(5) Kang, B.; Jang, M.; Chung, Y.; Kim, H.; Kwak, S. K.; Oh, J. H.; Cho, K. Enhancing 2D growth of organic semiconductor thin films with macroporous structures via a small-molecule heterointerface. Nat. Commun. 2014, 5, No. 4752.

(6) Zimmerman, J. D.; Xiao, X.; Renshaw, C. K.; Wang, S.; Diev, V. V.; Thompson, M. E.; Forrest, S. R. Independent control of bulk and interfacial morphologies of small molecular weight organic heterojunction solar cells. Nano Lett. 2012, 12, 4366-4371.

(7) Forker, R.; Fritz, T. Optical differential reflectance spectroscopy of ultrathin epitaxial organic films. Phys. Chem. Chem. Phys. 2009, 11, $2142-2155$.

(8) Dienel, T.; Loppacher, C.; Mannsfeld, S. C. B.; Forker, R.; Fritz, T. Growth-mode-induced narrowing of optical spectra of an organic adlayer. Adv. Mater. 2008, 20, 959-963.

(9) Müller, M.; Le Moal, E.; Scholz, R.; Sokolowski, M. Exciton and polarization contributions to optical transition energies in an epitaxial organic monolayer on a dielectric substrate. Phys. Rev. B 2011, 83, No. 241203.

(10) Müller, M.; Paulheim, A.; Marquardt, C.; Sokolowski, M. Spectroscopy of isolated PTCDA molecules on the $\mathrm{KCl}(100)$ surface: Vibrational spectra and azimuthal orientation. J. Chem. Phys. 2013, 138, No. 064703.
(11) Imada, H.; Miwa, K.; Imai-Imada, M.; Kawahara, S.; Kimura, K.; Kim, Y. Real-space investigation of energy transfer in heterogeneous molecular dimers. Nature 2016, 538, 364.

(12) Zhang, Y.; Luo, Y.; Zhang, Y.; Yu, Y.-J.; Kuang, Y.-M.; Zhang, L.; Meng, Q.-S.; Luo, Y.; Yang, J.-L.; Dong, Z.-C.; Hou, J. G. Visualizing coherent intermolecular dipole-dipole coupling in real space. Nature 2016, 531, 623.

(13) Korolkov, V. V.; Svatek, S. A.; Summerfield, A.; Kerfoot, J.; Yang, L.; Taniguchi, T.; Watanabe, K.; Champness, N. R.; Besley, N. A.; Beton, P. H. van der Waals-induced chromatic shifts in hydrogenbonded two-dimensional porphyrin arrays on boron nitride. ACS Nano 2015, 9, 10347-10355.

(14) Kerfoot, J.; Korolkov, V. V.; Nizovtsev, A. S.; Jones, R.; Taniguchi, T.; Watanabe, K.; Lesanovsky, I.; Olmos, B.; Besley, N. A.; Besley, E.; Beton, P. H. Substrate-induced shifts and screening in the fluorescence spectra of supramolecular adsorbed organic monolayers. J. Chem. Phys. 2018, 149, No. 054701.

(15) Alkhamisi, M.; Korolkov, V. V.; Nizovtsev, A. S.; Kerfoot, J.; Taniguchi, T.; Watanabe, K.; Besley, N. A.; Besley, E.; Beton, P. H. The growth and fluorescence of phthalocyanine monolayers, thin films and multilayers on hexagonal boron nitride. Chem. Commun. 2018, 54, 12021-12024.

(16) Mikhnenko, O. V.; Blom, P. W. M.; Nguyen, T.-Q. Exciton diffusion in organic semiconductors. Energy Environ. Sci. 2015, 8, $1867-1888$.

(17) Hiroshiba, N.; Morimoto, K.; Hayakawa, R.; Wakayama, Y.; Mori, T.; Matsuishi, K. Exciton dynamics at the heteromolecular interface between $\mathrm{N}, \mathrm{N}^{\prime}$-dioctyl-3,4,9,10-perylenedicarboximide and quaterrylene, studied using time-resolved photoluminescence. AIP Adv. 2014, 4, No. 067112.

(18) Lunt, R. R.; Giebink, N. C.; Belak, A. A.; Benziger, J. B.; Forrest, S. R. Exciton diffusion lengths of organic semiconductor thin films measured by spectrally resolved photoluminescence quenching. J. Appl. Phys. 2009, 105, No. 053711.

(19) Schüppel, R.; Dienel, T.; Leo, K.; Hoffmann, M. Time-resolved luminescence quenching in thin films of perylene-tetracarboxylicdianhydride. J. Lumin. 2004, 110, 309-314.

(20) Topple, J. M.; Burke, S. A.; Fostner, S.; Grütter, P. Thin film evolution: dewetting dynamics of a bimodal molecular system. Phys. Rev. B 2009, 79, No. 205414.

(21) Burke, S. A.; Topple, J. M.; Grütter, P. Molecular dewetting on insulators. J. Phys.: Condens. Matter 2009, 21, No. 423101.

(22) Körner, M.; Loske, F.; Einax, M.; Kühnle, A.; Reichling, M.; Maass, P. Second-layer induced island morphologies in thin-film growth of fullerenes. Phys. Rev. Lett. 2011, 107, No. 016101.

(23) Burke, S. A.; Mativetsky, J. M.; Fostner, S.; Grütter, P. $C_{60}$ on alkali halides: epitaxy and morphology studied by noncontact AFM. Phys. Rev. B 2007, 76, No. 035419.

(24) Burke, S. A.; Mativetsky, J. M.; Hoffmann, R.; Grütter, P. Nucleation and submonolayer growth of $\mathrm{C}_{60}$ on KBr. Phys. Rev. Lett. 2005, 94, No. 096102.

(25) Gaisch, R.; Berndt, R.; Gimzewski, J. K.; Reihl, B.; Schlittler, R. R.; Schneider, W. D.; Tschudy, M. Internal structure of $\mathrm{C}_{60}$ fullerence molecules as revealed by low-temperature STM. Appl. Phys. A 1993, 57, 207-210.

(26) Tanigaki, K.; Kuroshima, S.; Fujita, J. I.; Ebbesen, T. W. Crystal growth of $\mathrm{C}_{60}$ thin films on layered substrates. Appl. Phys. Lett. 1993, $63,2351-2353$.

(27) Jia, Q.; Hu, Z.-X.; Ji, W.; Burke, S. A.; Gao, H.-J.; Grütter, P.; Guo, H. Adsorption of PTCDA and $\mathrm{C}_{60}$ on $\mathrm{KBr}(001)$ : electrostatic interaction versus electronic hybridization. Phys. Chem. Chem. Phys. 2016, 18, 11008-11016.

(28) Lindner, R.; Rahe, P.; Kittelmann, M.; Gourdon, A.; Bechstein, R.; Kühnle, A. Substrate templating guides the photoinduced reaction of $\mathrm{C}_{60}$ on calcite. Angew. Chem., Int. Ed. 2014, 53, 7952-7955.

(29) Rahe, P.; Lindner, R.; Kittelmann, M.; Nimmrich, M.; Kühnle, A. From dewetting to wetting molecular layers: $\mathrm{C}_{60}$ on $\mathrm{CaCO}_{3}(104)$ as a case study. Phys. Chem. Chem. Phys. 2012, 14, 6544-6548. 
(30) Loske, F.; Lübbe, J.; Schütte, J.; Reichling, M.; Kühnle, A. Quantitative description of $\mathrm{C}_{60}$ diffusion on an insulating surface. Phys. Rev. B 2010, 82, No. 155428.

(31) Loske, F.; Bechstein, R.; Schütte, J.; Ostendorf, F.; Reichling, M.; Kühnle, A. Growth of ordered $\mathrm{C}_{60}$ islands on $\mathrm{TiO}_{2}$ (110). Nanotechnology 2009, 20, No. 065606.

(32) Ehrlich, G.; Hudda, F. G. Atomic view of surface self-diffusion: tungsten on tungsten. J. Chem. Phys. 1966, 44, 1039-1049.

(33) Schwoebel, R. L.; Shipsey, E. J. Step motion on crystal surfaces. J. Appl. Phys. 1966, 37, 3682-3686.

(34) Loske, F.; Reichling, M.; Kühnle, A. Deposition sequence determines morphology of $\mathrm{C}_{60}$ and 3,4,9,10-perylenetetracarboxylic diimide islands on $\mathrm{CaF}_{2}$ (111). Jpn. J. Appl. Phys. 2011, 50, No. 08LB07.

(35) Hoffmann, M.; Schmidt, K.; Fritz, T.; Hasche, T.; Agranovich, V. M.; Leo, K. The lowest energy Frenkel and charge-transfer excitons in quasi-one-dimensional structures: application to MePTCDI and PTCDA crystals. Chem. Phys. 2000, 258, 73-96.

(36) Wewer, M.; Stienkemeier, F. Laser-induced fluorescence spectroscopy of $\mathrm{N}, \mathrm{N}^{\prime}$-dimethyl 3,4,9,10-perylene tetracarboxylic diimide monomers and oligomers attached to helium nanodroplets. Phys. Chem. Chem. Phys. 2005, 7, 1171-1175.

(37) Brabec, C. J.; Zerza, G.; Cerullo, G.; De Silvestri, S.; Luzzati, S.; Hummelen, J. C.; Sariciftci, S. Tracing photoinduced electron transfer process in conjugated polymer/fullerene bulk heterojunctions in real time. Chem. Phys. Lett. 2001, 340, 232-236.

(38) Chiş, V.; Mile, G.; Ştiufiuc, R.; Leopold, N.; Oltean, M. Vibrational and electronic structure of PTCDI and melaminePTCDI complexes. J. Mol. Struct. 2009, 924-926, 47-53.

(39) Gündüz, B.; Kurban, M. Photonic, spectroscopic properties and electronic structure of PTCDI-C8 organic nanostructure. Vib. Spectrosc. 2018, 96, 46-51.

(40) Beu, T. A.; Onoe, J.; Hida, A. First-principles calculations of the electronic structure of one-dimensional $\mathrm{C}_{60}$ polymers. Phys. Rev. B 2005, 72, No. 155416.

(41) Mutolo, K. L.; Mayo, E. I.; Rand, B. P.; Forrest, S. R.; Thompson, M. E. Enhanced open-circuit voltage in subphthalocyanine $/ \mathrm{C}_{60}$ organic photovoltaic cells. J. Am. Chem. Soc. 2006, 128, $8108-8109$. 